\title{
Phosphatidylinositol 3-kinase (PI3K) pathway activation in bladder cancer
}

\author{
Margaret A. Knowles $\cdot$ Fiona M. Platt • \\ Rebecca L. Ross • Carolyn D. Hurst
}

Published online: 16 December 2009

(C) The Author(s) 2009. This article is published with open access at Springerlink.com

\begin{abstract}
The phosphatidylinositol 3-kinase (PI3K) pathway is a critical signal transduction pathway that regulates multiple cellular functions. Aberrant activation of this pathway has been identified in a wide range of cancers. Several pathway components including AKT, PI3K and mTOR represent potential therapeutic targets and many small molecule inhibitors are in development or early clinical trials. The complex regulation of the pathway, together with the multiple mechanisms by which it can be activated, make this a highly challenging pathway to target. For successful inhibition, detailed molecular information on individual tumours will be required and it is already clear that different tumour types show distinct combinations of alterations. Recent results have identified alterations in pathway components PIK3CA, PTEN, AKT1 and TSC1 in bladder cancer, some of which are significantly related to tumour phenotype and clinical behaviour. Co-existence of alterations to several PI3K pathway genes in some bladder tumours indicates that these proteins may have functions that are not related solely to the known canonical pathway.
\end{abstract}

Keywords Bladder cancer PI3K pathway $\cdot$ PTEN . PIK3CA · AKT1 - TSC1

M. A. Knowles $(\varangle) \cdot$ F. M. Platt $\cdot$ R. L. Ross $\cdot$ C. D. Hurst

Cancer Research UK Clinical Centre,

Leeds Institute of Molecular Medicine,

St James's University Hospital,

Beckett Street,

Leeds LS9 7TF, UK

e-mail: M.A.Knowles@leeds.ac.uk

\section{Introduction}

The phosphatidylinositol 3-kinase (PI3K) pathway (Fig. 1) plays a pivotal role in cell growth, proliferation and survival [1] and signalling via this pathway is upregulated in many types of cancer $[2,3]$. Genomic alterations have been detected in multiple genes in the pathway. These include the tumour suppressor genes PTEN and LKB1, which are inactivated by mutation and/or deletion in many sporadic cancers $[4,5]$ and the protooncogenes $A K T 1$, $A K T 2, P D K 1$ and PIK3CA that are activated by mutation or amplification [6-9]. Other mutations and alterations in gene expression in cancer also contribute to aberrant activation of the pathway. For example, oncogenic activation of the RAS genes by point mutation activates the pathway via the interaction of RAS with $\mathrm{p} 110 \alpha$, the catalytic subunit of PI3 kinase [10]. Increased signalling via the epidermal growth factor family (ERBB) receptors also activates the pathway. The mTOR pathway can also be activated downstream of RAS via ERK-mediated negative regulation of the TSC1/ TSC2 complex [11]. Several of the activated protooncogenes represent potential targets for therapy and there is currently much interest in developing agents to target these proteins.

The mechanisms by which the pathway is activated are somewhat tissue specific. For example, PTEN is inactivated predominantly by mutation in certain cancer types but in others the major mechanism of inactivation is genomic homozygous deletion (HD) [4]. Some tumour types show mutual exclusivity of different events in the pathway and others do not [12]. Thus, for rational application of PI3K pathway-targeted agents, comprehensive information on pathway activation status and mechanisms of activation is needed for specific tumour types. Several mechanisms have been identified by which the PI3K pathway is activated in 


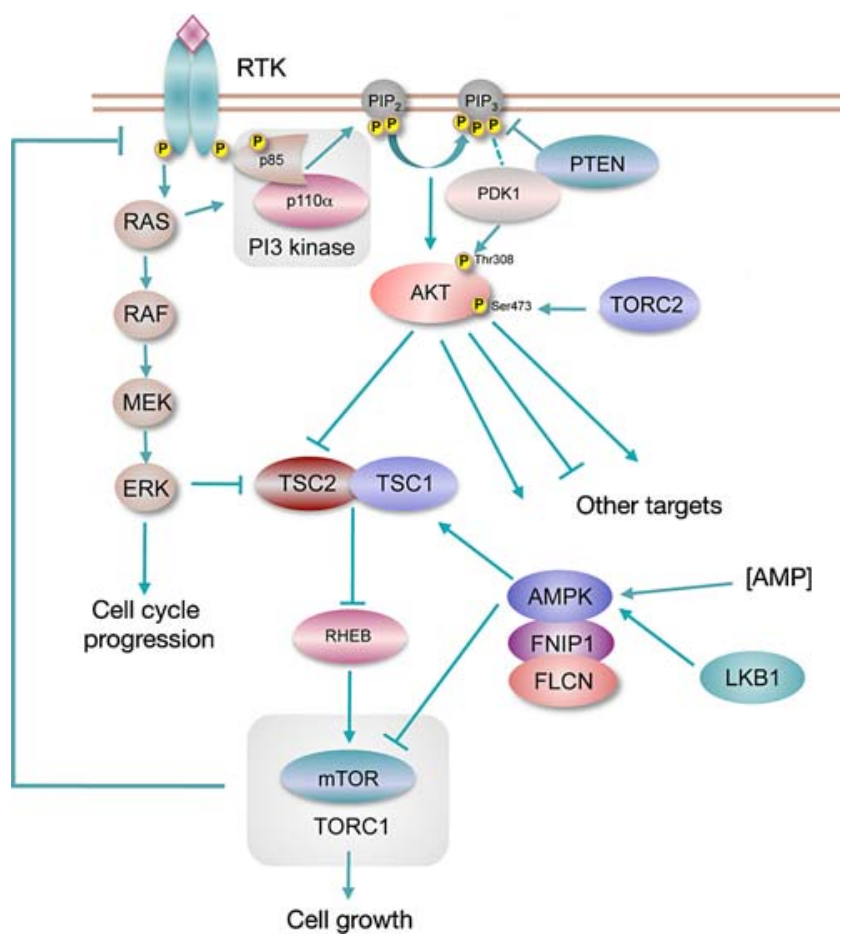

Fig. 1 The class IA PI3K signalling pathway

urothelial carcinoma (UC). This review will summarise recent findings and relate these to the major UC subtypes and potential therapeutic approaches.

\section{Bladder cancer subtypes}

Bladder cancers fall into two major groups with distinct pathology and clinical behaviour and different molecular profiles [13-15]. Non-invasive papillary tumours and muscle-invasive tumours represent distinct groups that at least in the majority of cases, do not represent a developmental continuum [13, 15]. Muscle-invasive bladder cancer (T2-T4) is thought to develop via flat dysplasia and carcinoma in situ (CIS) and these tumours are usually of non-papillary architecture. Non-invasive (Ta) tumours are believed to arise via flat urothelial hyperplasia followed by development of an exophytic papillary architecture. These represent the two ends of a spectrum within which lie the group of $\mathrm{T} 1$ papillary tumours that invade the submucosa but not muscle. For T1 tumours, diagnosis and prediction of clinical course are difficult and it is not clear how the pathogenesis of this group relates to that of the Ta and muscle invasive tumours (T2-4). Figure 2 shows the proposed pathogenesis of the two major groups of bladder tumours and possible relationships to $\mathrm{T} 1$ disease. A hypothetical third pathogenesis pathway involving initial flat dysplasia leading to the development of high-grade papillary tumours is included though evidence for this is currently lacking. Papillary T1 tumours could develop via this route.

Recent molecular studies have implicated PI3K pathway alterations in UC of all grades and stages. However, some associations with particular groups have been identified and will be discussed below. It is clear that for full biological understanding and rational application of therapeutic agents, precise information on the timing and pathological associations of these molecular alterations and their interactions and/or co-operation with other events will be required.

\section{PI3K pathway}

PI3 kinases are heterodimers comprised of a regulatory subunit (p85) and a catalytic subunit (p110). Activated receptor tyrosine kinases recruit the PI3 kinase complex to the membrane via the $\mathrm{p} 85$ regulatory subunit either directly or via insulin receptor substrate adapter proteins, activating the catalytic subunit p110, which then phosphorylates phosphatidylinositol-4,5-bisphosphate (PIP2) to phosphatidylinositol-3,4,5-trisphosphate (PIP3). Phosphatase and tensin homologue deleted on chromosome 10 (PTEN) catalyses the reverse reaction. PIP3 recruits protein dependent kinase 1 (PDK1) and AKT, also known as protein kinase $\mathrm{B}(\mathrm{PKB})$, to the plasma membrane where AKT is phosphorylated at Thr308 by PDK1 and at Ser473 by mTOR complex 2 (TORC2) [16]. AKT1 can also be phosphorylated at Ser473 by DNA-PK in the nucleus [17]. AKT occupies a key regulatory node in the PI3K pathway, below which the pathway branches significantly to influence a wide range of cellular processes that promote cell cycle progression, cell growth, energy metabolism and resistance to apoptosis. One of the major effectors downstream of AKT is mTOR complex 1 (TORC1), which integrates inputs related to growth-factor signalling and nutrient and oxygen availability to regulate protein synthesis (Fig. 1).

In bladder cancer, mutations have been identified in p110 $\alpha$ (PIK3CA), AKT1, TSC1 and loss of heterozygosity $(\mathrm{LOH})$, homozygous deletion and inactivating mutations of PTEN have been found [18-21].

\subsection{PIK3CA}

There are three isoforms of class IA p110 (p110 $\alpha$, p110 $\beta$ and $\mathrm{p} 110 \delta$ ). All have similar structure with domains for binding to the adaptor protein PIK3R1 (p85) and to RAS (Fig. 3a). To date, most evidence from human cancers implicates $\mathrm{p} 110 \alpha$ (PIK3CA) with activating mutations and/ or amplification identified in a wide range of tumour types [22]. 
Fig. 2 Potential pathways of urothelial tumorigenesis. Solid arrows indicate likely pathways and broken arrows indicate uncertain relationships. Low-grade papillary tumours (left) may arise via simple hyperplasia and minimal dysplasia. Invasive carcinoma (right) is believed to arise via the flat high-grade lesion CIS. A third hypothetical pathway to development of high grade papillary tumours is shown (middle). The pathway to development of $\mathrm{T} 1$ tumours is uncertain

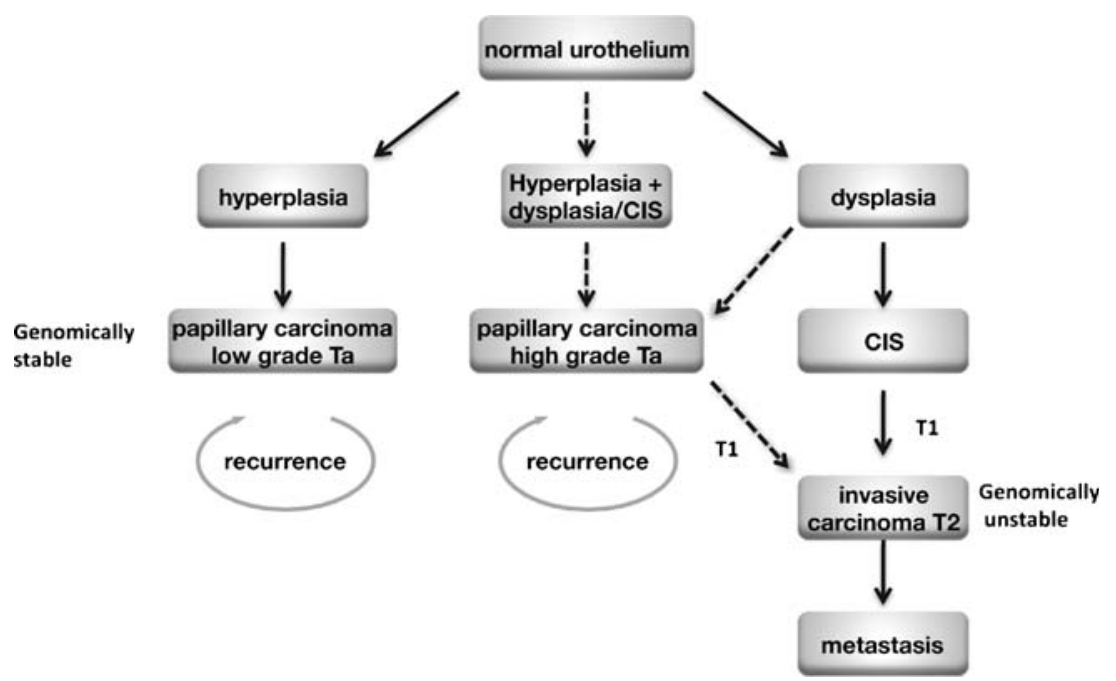

In UC, mutation of PIK3CA has been found in a significant proportion of tumours, including many $\mathrm{Ta}$ tumours. The overall frequency in a series of tumours comprising $35.4 \% \mathrm{Ta}, 39.2 \% \mathrm{~T} 1$ and $25.4 \% \mathrm{~T} 2$ tumours was $27 \%$ [23]. In a study that examined a larger proportion of Ta tumours $(65.5 \% \mathrm{Ta}, 11.5 \% \mathrm{Ta}, 23 \% \mathrm{~T} 2)$, a significant association of mutation with low grade and stage was found [24], though overall frequency was lower (13\%). The difference in frequency in these studies may reflect the screening of only hotspot exons in the study of LopezKnowles et al., although this is unlikely as only two mutations outside these hotspot regions were identified in the first study. Possibly, the difference may be attributed to differences in sensitivity of the mutation-screening approaches used (direct sequencing vs. scanning by highresolution melting followed by sequencing). Further studies are needed to clarify the exact frequency and distribution of these mutations according to tumour grade and stage. The recent development of a low-cost, high throughput assay to detect the common variants should facilitate this [25].

Interestingly, the PIK3CA mutation spectrum in $\mathrm{UC}$ differs significantly from that in other cancers. Figure $3 b$ shows the spectrum of mutations in UC [23] and that reported in COSMIC (www.sanger.ac.uk/genetics/CGP/ cosmic/). Mutations E542K and E545K in the helical domain are most common and the kinase domain mutation H1047R which is the most common mutation in other cancers is less common in UC. In total, 9 different mutations have been found to date [23] (Fig. 3a). Mutations resulting in amino acid changes P124L, Q643R, D1017H and M1043I were confirmed as somatic by comparison with matched normal DNA. The mutation A1066V found in the cell line $639 \mathrm{~V}$ could not be confirmed as somatic as paired normal DNA is not available for this cell line. Apart
Fig. 3 Mutations of $P I K 3 C A$ identified in bladder cancer. a Schematic representation of the protein showing functional domains and the position of mutations identified to date. Both the adapter-binding and $\mathrm{C} 2$ domains interact with $\mathrm{p} 85$. b Comparison of PIK3CA mutations in bladder cancer and those listed in COSMIC for positions M1043, H1047, E542 and E545 excluding bladder tumour mutations modified from [23]

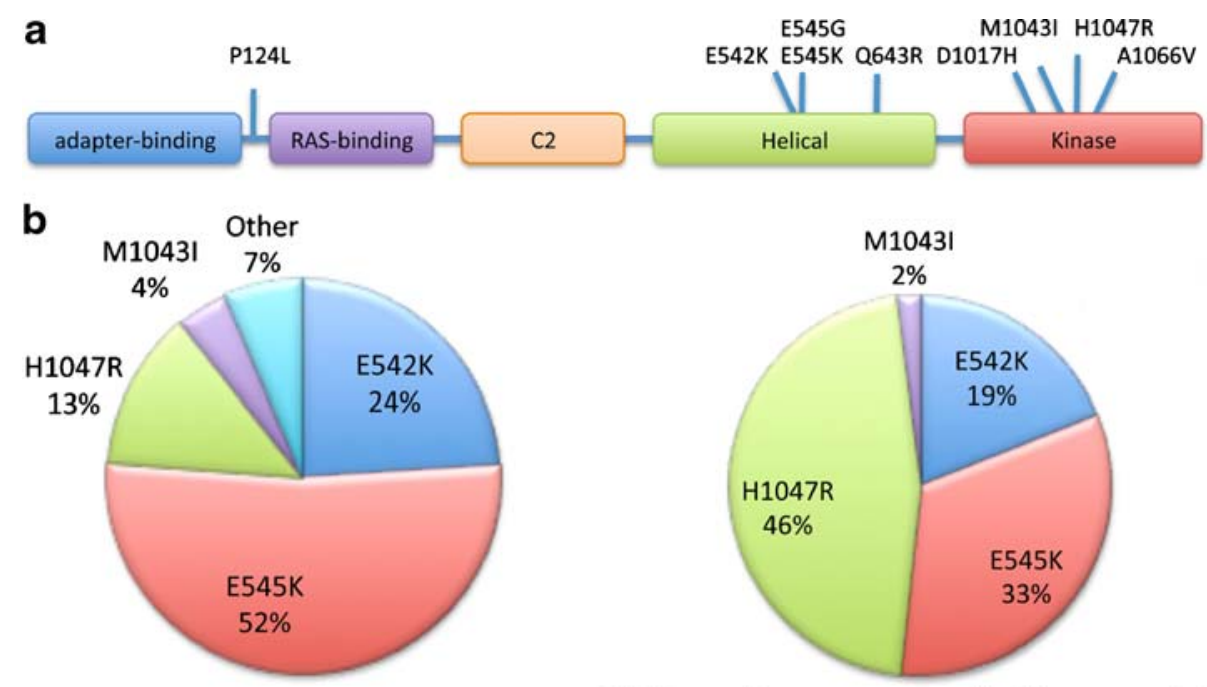

PIK3CA mutation spectrum: bladder (Platt et al., 2009)
PIK3CA mutation spectrum:non-bladder tumors in COSMIC 
from $\mathrm{P} 124 \mathrm{~L}$, these rare mutations are all close to known hotspot mutations and therefore predicted to affect protein function in similar ways. P124L lies in a region of four helices in the protein between the adapter-binding and RAS-binding domains, the function of which is not known. However, a missense mutation in codon 124 (P124T) has been reported in a colorectal cancer [26] suggesting that this may be an activating mutation. Functional studies are now required to confirm this.

The selective pressure for helical domain mutation in UC is not yet understood. Recent studies of the structure of p110 $\alpha$ and the effect of the common activating mutations have revealed different mechanisms of activation by helical domain and kinase domain mutations [22]. In functional assays, H1047R was shown to be dependent on p85 binding but not on RAS binding and, conversely, the helical domain mutations E542K and E545K were shown to require RAS binding but not $\mathrm{p} 85$ binding. This raises the possibility that there may be co-operation between these helical domain PIK3CA mutant proteins and other events in UC that are known to activate RAS including mutations of the RAS genes themselves [27], epidermal growth factor receptor (EGFR) overexpression and FGFR3 mutation [28]. It is interesting that mutations in FGFR 3 and PIK3CA are also found commonly in the benign skin lesions seborrheic keratosis and epidermal nevi [29]. As many Ta papillary bladder tumours can be considered benign, these molecular alterations may be hallmarks of benign epithelial overgrowth conditions.

\subsection{PTEN}

PTEN is a multifunctional phosphatase whose major lipid substrate is PIP3 generated at the cell membrane by PI3K. PTEN can also dephosphorylate a range of protein targets including She and FAK [30]. PTEN is found not only at the cell membrane and in the cytoplasm but also in the nucleus where it is believed to exert additional tumour-suppressive functions (reviewed in [31]). Deletions of 10q including the PTEN region are found in $24-58 \%$ of invasive UC $[18,20$, 32 and only infrequently in low-grade papillary noninvasive UC. In contrast to some other tumour types, mutations on the retained allele of PTEN are not detected frequently in UC [19-21,33], but HD has been identified in a few cases.

Recent studies have shown that reduced PTEN protein expression is common in UC $[23,34,35]$. PTEN is haploinsufficient in mouse models [36] and findings in UC to date are compatible with a phenotypic effect of reduced expression in the urothelium. Downregulation of PTEN expression is more frequent in invasive UC and is commonly associated with p53 alteration. Thus, $41 \%$ of tumours with altered p53 also showed downregulation of
PTEN and this combined alteration of p53 and PTEN was associated with poor patient outcome [35]. To date, only a single study has examined pathway activation status in UC. Western blot analysis of levels of phosphorylated AKT revealed that $55 \%$ had significantly higher levels than normal controls. These included five of 11 superficial tumours and six of 11 invasive tumours [37].

Based on the observation that PTEN functions in both nucleus and cytoplasm, two studies have examined nuclear and cytoplasmic PTEN expression separately [23, 34]. Results were concordant, each study showing that nuclear staining was reduced more frequently than cytoplasmic staining [23, 34]. Reduced PTEN expression was significantly associated with tumour grade, stage or both. Figure 4 shows examples of PTEN protein expression patterns in UC and Fig. 5 shows the distribution of nuclear and cytoplasmic expression according to grade and stage in our recent study [23].

UC cell lines have also been assessed for allelic status of $P T E N$, copy number reduction by array $\mathrm{CGH}$ and protein expression. Several lines have homozygous deletion of PTEN (J82, UM-UC3, DSH1) and two (T24 and 639 V) are known to contain missense mutations. Overall, $46 \%$ of UC lines examined recently had alterations that implicated PTEN [23]. As all but one of these lines was derived from invasive UC, these findings are compatible with what has been observed in muscle invasive bladder tumour tissues.

The protein phosphatase activity of PTEN influences cell motility via its action on proteins involved in cytoskeletal re-modelling. In glioma, protein phosphorylation activity of PTEN controls FYN kinase to regulate cell migration in a PI3K pathway-independent manner [38]. It has been shown previously that the germline mutation of PTEN, G129E, which is found in Cowden syndrome, results in loss of the lipid phosphatase activity of the protein but not its protein phosphatase activity. This has led to a major focus on the lipid phosphatase activity of PTEN and less focus on the consequences of its activity as a protein phosphatase.

A critical role for activation of the PI3K pathway in UC cell lines has been demonstrated by the finding that the PI3K inhibitor LY294002, but not a MEK1/2, inhibitor suppressed the invasive properties of T24, J82 and UMUC3 cell lines, all of which have PTEN defects [37]. Reexpression of wild-type PTEN or expression of a dominant negative AKT also suppressed invasion, confirming that these cells are dependent on activation of the PI3K pathway for their invasive phenotype. This effect of PTEN reexpression in J82 was subsequently confirmed in an independent study [39]. The G129E mutant form of PTEN has been used to demonstrate that the invasive phenotype of T24, which contains a mutation in PTEN, can be inhibited by the protein phosphatase activity of PTEN alone [40]. This phenotype is likely to be of critical importance in the 


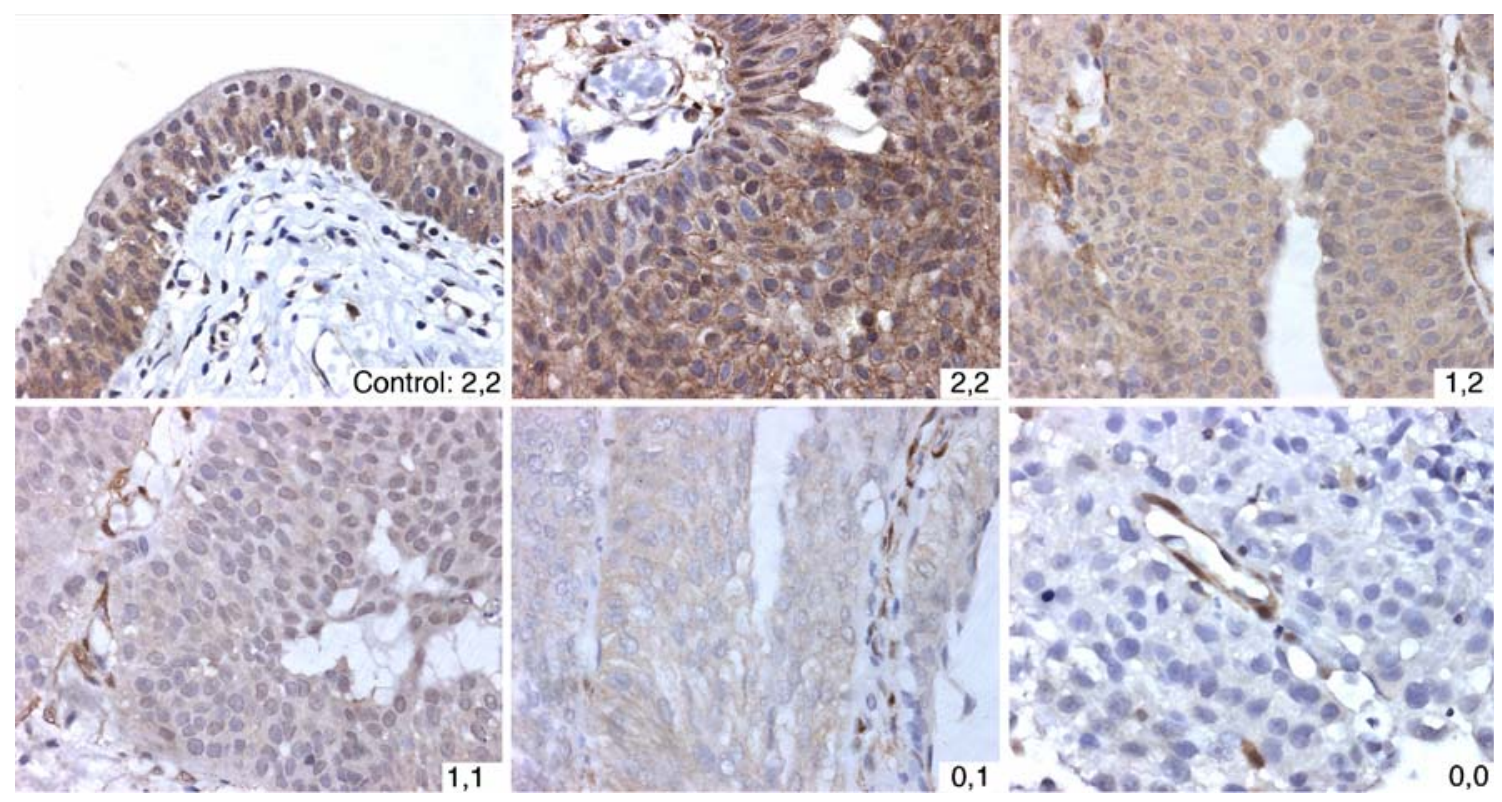

Fig. 4 Patterns of staining for PTEN protein in urothelial tumours. Scores are nuclear followed by cytoplasmic intensity. Normal ureter is shown as control. Staining in tumour samples was scored as 2 if it was at least as strong as the staining in controls, 1 if detectable but reduced and 0 if staining was absent. In tumour samples with scores of 1,2 ; 1,$1 ; 0,1 ; 0,0$ stromal cells with intense staining act as positive controls. All images were captured at same magnification. Reprinted from [23]

canonical activities of PTEN, possibly via its protein phosphatase activity, in invasive UC. Further studies are required to understand exactly how these two enzymatic functions of PTEN play a role in the two major groups of UC.
Fig. 5 Distribution of nuclear and cytoplasmic staining intensities for PTEN according to bladder tumour grade and stage. Reprinted from [23]
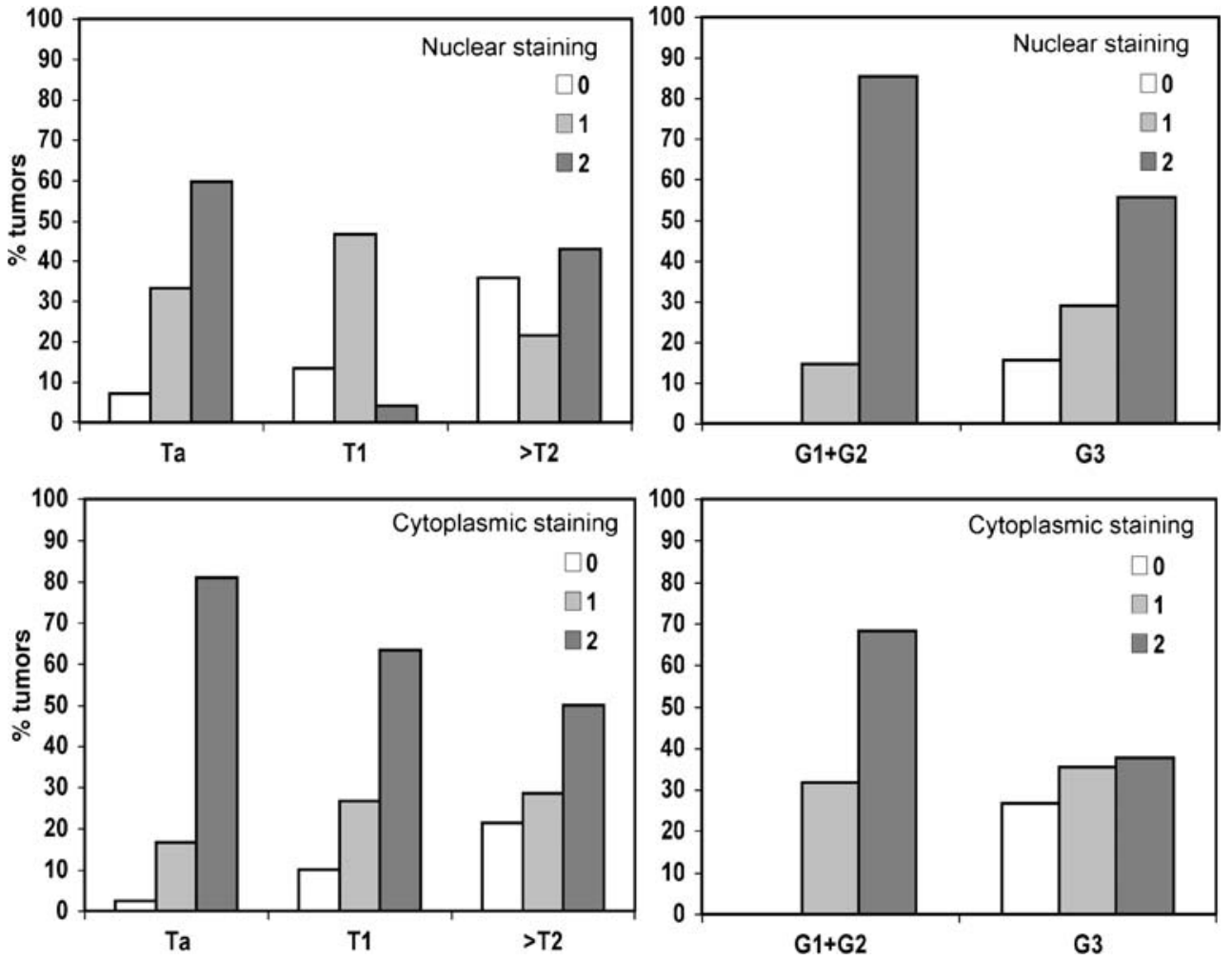
Several studies have examined the contribution of PTEN loss to bladder cancer development in mouse models [35]. Conditional deletion of Pten in the urothelium led to early urothelial hyperplasia [34, 41]. Increased cell size was observed, compatible with mTOR activation, and PI3K pathway activation was confirmed by detection of phosphoAKT [34]. UC developed late in these animals and was typically pedicellate papillary transitional cell carcinoma, resembling human papillary superficial tumours. Development of these tumours could be precipitated by treatment with the known bladder carcinogen butyl- $N$-(4-hydroxybutyl)nitrosamine. A more recent study used adenovirusdelivered Cre recombinase to induce stochastic deletion of p53 and/or Pten in mice carrying floxed alleles [35]. Deletion of either gene alone did not lead to tumour formation but dual deletion led to the early development of aggressive tumours, which frequently metastasised, providing a model of aggressive human UC with inactivation of both of these tumour suppressor genes.

\section{$3.3 \mathrm{AKT} 1$}

AKT1 is one of three family members of serine-threonine kinases that are activated by phosphorylation of Thr308 by PDK1 and Ser473 by TORC2 (Fig. 1). Recently, an activating mutation in the pleckstrin homology domain of AKT1 was identified in breast, colorectal and ovarian cancers [9]. Glu 17 plays a pivotal role in activation of AKT1 due to its location in the phosphoinositide-binding pocket. The change to lysine introduces a positive change that alters phosphoinositide affinity and the mutant protein is aberrantly translocated to the membrane resulting in constitutive downstream signalling.

The same mutation has been identified in other cancers including bladder [42]. G49A (E17K) was found in two of $44(4.8 \%)$ bladder cancer cell lines and five of $184(2.7 \%)$ UC tumour samples. In cell lines containing the mutation, AKT1 activation was retained under conditions of growth factor withdrawal. A novel mutation, G145A (E49K), was also found in one cell line. This mutation also enhanced AKT activation and was transforming in NIH3T3 cells. Functional studies using retroviruses to deliver wild-type AKT1 or the two mutants singly or in tandem, indicated enhanced activation of AKT1 when E17K and E49K mutations were in tandem suggesting that they can cooperate. However, the E49K mutation was not found in a series of $184 \mathrm{UC}$ tumour samples indicating that it does not contribute to the development of the majority of UC.

\section{$3.4 \mathrm{TSC} 1$}

TSC1 is one of two genes that when mutated in the germline cause the syndrome tuberous sclerosis complex
(TSC), an autosomal dominant tumour-suppressor gene syndrome characterised by the development of hamartomas in the kidneys, heart, brain and skin [43]. TSC1 on chromosome 9q34 encodes hamartin [44] and TSC2 on chromosome 16p13.3 encodes tuberin [45]. Tumour development in TSC patients is thought to occur as the result of a somatic 'second-hit' in one of these genes.

Co-localisation and co-immunoprecipitation of TSC1 and TSC2 in mammalian cells [46, 47] and binding in yeast two-hybrid assays, provides evidence for direct interaction of the two proteins and a rationale for the similar disease phenotype in patients with mutations in either gene $[44,48]$. Functionally, the TSC1/TSC2 complex is positioned downstream of AKT where TSC2 is directly phosphorylated and inactivated by AKT. Activation of the complex in growth-limiting conditions attenuates signalling through mTOR via specific GTPase activating protein activity of TSC2 towards the small GTPase RHEB [49, 50]. Inactivation of the complex leads to enhanced signalling via mTOR.

Although epithelial malignancy is not a common feature of TSC, loss of function of TSC1 has been identified in UC $[23,51,52]$. To date, no other human malignancies have been shown to contain mutations in either of the TSC genes. $\mathrm{LOH}$ for markers on chromosome nine is observed in more than $50 \%$ of bladder tumours of all grades and stages [53] and sub-chromosomal LOH analyses have identified the TSC1 locus at $9 \mathrm{q} 34$ as a common critical region of deletion between markers D9S149 and D9S66 $[51,54-56]$. Studies to date indicate an overall TSC1 mutation frequency of $14.5 \%$ in UC. The mutation spectrum comprises nonsense (35\%), missense (26\%), frameshift $(26 \%)$, in-frame deletions $(3 \%)$ and splicing $(10 \%)$ mutations $[23,57]$ (Fig. 6). Missense mutations of TSC1 found in UC were shown to cause loss of function through aberrant splicing, protein instability or protein mislocalisation [58]. More recently, it has become apparent that such mechanisms of inactivation via missense mutation are also found in TSC disease [59, 60].

It has been suggested that TSCl is haploinsufficient in some lesions in patients with germline mutation [61] and we previously found apparent heterozygosity for mutations in UC tumour samples [57]. Recently, we analysed a series of tumours more carefully to assess possible haploinsufficiency and found only one case with mutation in which the wild-type allele of TSC1 was retained. This tumour contained the missense mutation F216A and continued to express hamartin [23]. Previously, we have shown altered localisation of this mutant protein when re-expressed in TSC1-null urothelial cells and unlike other somatic missense mutants, there is no splicing defect or reduced protein stability [58] pointing to altered function (possibly dominant negative) rather than haploinsufficiency in this case. 


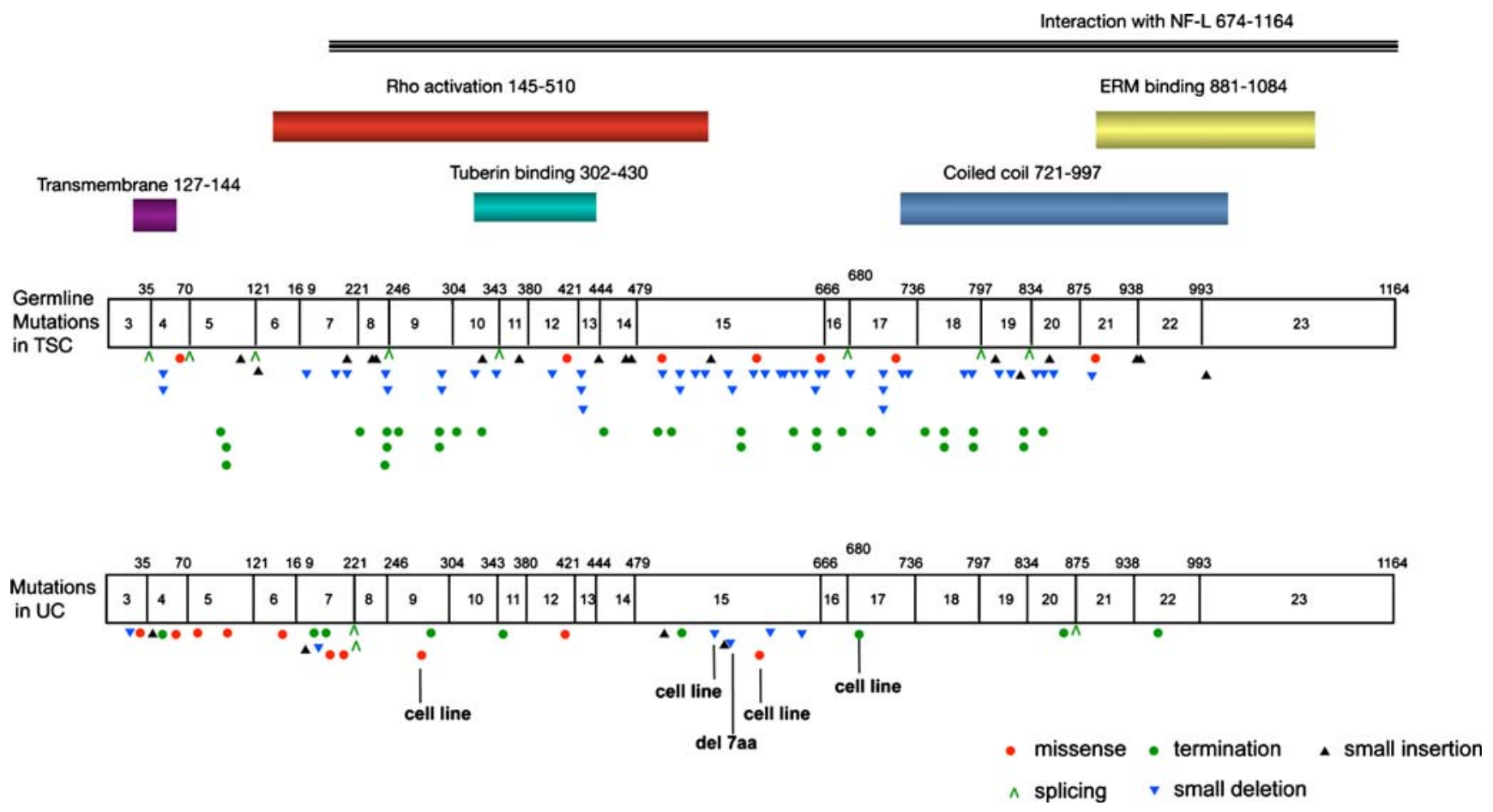

Fig. 6 TSC1 mutation spectrum in tuberous sclerosis complex and in bladder cancer. Amino acid positions of reported functional domains of hamartin are shown

Immunohistochemical analysis of hamartin expression in UC tissues does, however, reveal a range of levels of protein expression in tumours that do not carry a mutation (Fig. 7) indicating that other mechanisms of regulation of expression may play a role in reducing TSC1 function in UC.

As tuberin represents the active partner in the TSC1/ TSC2 complex, somatic inactivation of TSC2 could have more profound effects than inactivation of TSC1. Indeed, germline TSC2 mutations cause a more severe phenotype than TSC1 mutations [62]. TSC2 is a large gene and has not been screened for mutation in UC. $\mathrm{LOH}$ analysis of the TSC2 gene region has revealed only $15 \% \mathrm{LOH}$ and arraybased CGH analysis has shown underrepresentation of the TSC2 region in only $9.7 \%$ of cases indicating that biallelic inactivation of TSC2 is unlikely to be common in UC [23].

\subsection{Upstream activators of the PI3 kinase pathway}

The pathway is activated by a range of receptor tyrosine kinases. These include the ERBB family of receptors, EGFR, ERBB2, ERBB3 and ERBB4. ERBB3 interacts

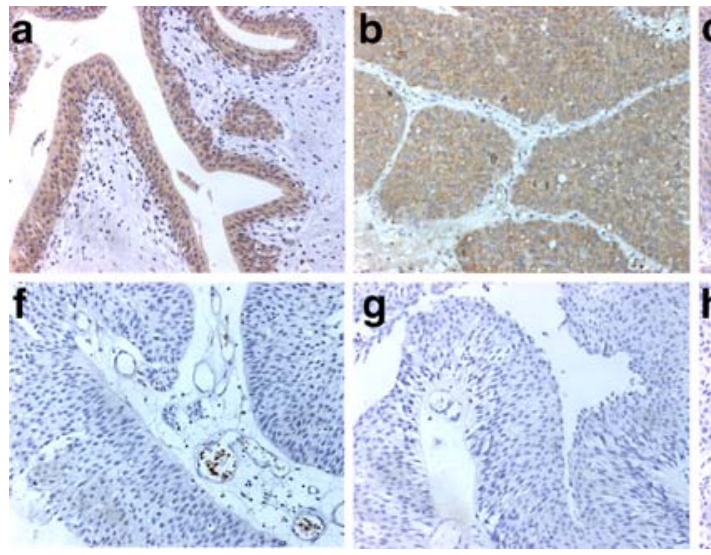

Fig. 7 Patterns of staining for the TSC1 protein product hamartin in bladder tumours. a Normal ureter. b-j Bladder tumour tissues. b, c, d and $\mathbf{e}$ illustrate the range of staining intensity in positive-staining tumours. f-j show loss of hamartin expression. In i, the tumour shows loss of expression and a region of normal urothelium is present at the bottom of the image. Reprinted from [23] 
directly with $\mathrm{p} 110 \alpha$ [63], whilst EGFR induces activation via RAS. Although there has been some conflicting evidence, most studies of UC report over-expression of EGFR, ERBB2 and/or ERBB3 in subsets of cases (e.g., [64-69]). High-level expression of EGFR is associated with higher tumour grade and stage and with decreased recurrence-free interval [70]. ERBB2 and ERBB3 expression is associated with high tumour grade and stage, though it is not clear whether this provides predictive or prognostic information [71, 72]. ERBB4 has been reported to show reduced expression in tumours compared with normal urothelium [70]. Further information is needed on the expression of these receptors and their ligands in UC but it is clear that activation of the PI3 kinase pathway in many advanced cases of UC may be driven by one of more members of this family.

Other receptor tyrosine kinases that are known to activate the pathway include KIT, RON (MST1R), PDGFRA and MET. There is no information on the expression of KIT or PDGFRA in UC. However, MET and RON expression is upregulated in aggressive UC [7375]. Expression status of these proteins has not yet been examined in relation to PI3K pathway activation status.

$\mathrm{N}$-cadherin is upregulated in UC, mainly in invasive tumours [76], and it has been shown that ectopic expression of this cadherin in UC cell lines induces cell invasion [77]. This was associated with activation of the PI3K pathway and was inhibited by both PI3K and SRC inhibitors. The exact mechanism by which $\mathrm{N}$-cadherin activates the pathway remains to be elucidated.

RAS proteins interact directly with $\mathrm{p} 110 \alpha$. Activating mutations in one of the three RAS genes are found in $13 \%$ of UC [27]. These are predominantly in HRAS and KRAS ([27]; Hurst et al., unpublished), but a few NRAS mutations have also been identified. There appears to be no association of mutation with clinico-pathological parameters. It is predicted that the PI3K pathway is activated directly by RAS in these tumours.

Finally, it is possible that activating mutation or overexpression of FGFR3 can activate the pathway in UC. RAS and FGFR3 mutations are mutually exclusive in these tumours, suggesting that they may fulfil overlapping functions [27]. Mutant FGFR3 has been confirmed to activate the RAS-MAPK pathway in normal human urothelial cells, though in this normal cell context, activation of the PI3K pathway was not detected [78]. It has been observed that although RAS and FGFR3 mutation are mutually exclusive, the grade and stage distribution of these events is different, FGFR3 mutations being most common in low-grade Ta tumours and RAS mutations being distributed evenly across tumours of all grades and stages. This could indicate differences in downstream effector mechanisms and PI3K pathway activation is a candidate for such a difference. It will be important to examine the effects of FGFR3 in the context of urothelial tumour cells rather than normal cells and to explore the pathway activation status and clinical outcome of UC with RAS mutation in comparison with those of similar grade and stage with FGFR3 mutation.

\subsection{Other genes in the pathway}

It is possible that other genes in the pathway are altered in UC. To date, screening of only $L K B 1$ and RHEB has been reported [23]. No mutations were found in $R H E B$.

$L K B 1$ is a serine threonine kinase that activates AMPactivated protein kinase [79] and negatively regulates mTOR signalling via TSC1/TSC2 [80]. Germline mutation of LKB1 causes Peutz-Jegher's syndrome, a multiple hamartoma syndrome with a range of benign tumours including a few in the ureter and bladder [81]. In UC, only a single somatic mutation, a missense change (Q100E) was found, indicating that $L K B 1$ is not a critical mutation target in UC unlike other sporadic cancers [82]. The functional significance of this missense change is unknown. In other tumour types, however, the majority of mutations reported are protein-truncating (stop or frameshift).

Other candidate genes that merit examination are PIK3R1 (p85), mutations of which have been reported at low frequency in other epithelial cancers [6], AKT2, AKT3, PDK1 and FLCN, which when mutated in the germline causes Birt-Hogg-Dubé syndrome, characterised by benign skin, kidney and lung lesions [83].

\subsection{Relationship of pathway alterations}

If the PI3K pathway is considered as a linear pathway, it might be predicted that alterations affecting any event in the pathway would have the same biological consequences and thus would be redundant and mutually exclusive in cancer. In some tumour types, this does indeed appear to be the case. Thus for example, there is virtual mutual exclusivity of RAS and PIK3CA mutation in endometrial cancer. However, these mutations co-exist in colorectal cancer, and in breast cancer HER2 amplification, PIK3CA mutation and PTEN loss co-exist (reviewed in [12]).

In UC, genomic alterations in three of the key genes in the pathway (PIK3CA, TSC1, PTEN) are not mutually exclusive [23], implying that combined mutations have additive or synergistic effects and that non-canonical effects of these components may be critical drivers of bladder cancer. This may be particularly important for TSC1. The widely studied functions of both TSC1 and TSC2 are attributed to the TSC1/TSC2 complex that regulates mTOR activity via RHEB. Although some independent functions have been ascribed to TSC2, independent function of TSC1 
is not clear. The finding of mutations in bladder but not other cancers and the lack of mutual exclusivity of TSC1 mutations with either PIK3CA or PTEN alterations may indicate that TSC1 has an independent function in the urothelium.

\section{Therapeutic implications}

The prominent role of the PI3K pathway in a wide range of solid malignancies has led to major efforts to develop agents that target components of the pathway. Discussion of the wide range of inhibitors and approaches in development is beyond the scope of this review and only a brief comment will be given here. Two excellent recent reviews provide detailed descriptions [84, 85].

Although there are currently no active clinical studies of PI3K pathway inhibitors in UC, it is clear that these tumours may benefit greatly from such agents. Several classes of inhibitors may be relevant; dual PI3K-mTOR inhibitors, PI3K inhibitors, AKT inhibitors and mTOR inhibitors. Dual inhibitors of PI3K and mTOR reflect the similarity in structure of these proteins, which has resulted in the identification of small molecules that target both. These typically target $\mathrm{p} 110 \alpha, \beta$ and $\delta$ isoforms, TORC1 and TORC2. This broad effect should potently inhibit PI3K-AKT-TORC1 signalling. PI3K inhibitors are either pan-PI3K or isoform specific. Pan inhibitors show side effects including immunosuppression and glucose intolerance. There is the theoretical advantage with isoformspecific agents that these unwanted effects might be avoided and, in cancers with specific dependence on a single isoform, this approach may be preferable. However, there are no p110 $\alpha$-specific agents yet in clinical trials. In UC, where tumours show alterations at many levels in the pathway, combined PI3K-mTOR inhibition may be a useful approach, though may be relatively toxic.

As activation of mTOR can also be accomplished via the MAPK pathway [11] (Fig. 1) it is likely that in tumours with activation of this pathway in addition to the PI3K pathway, inhibition of TORC1 cannot be achieved via inhibition of the PI3K pathway alone. Similarly, tumours with AKT1 mutation would not be predicted to respond to PI3K inhibitors. In these cases, the specific AKT inhibitors currently in phase I studies may be appropriate.

Rapamycin and its analogues inhibit TORC1 but not TORC2. These have yet to be tested in UC but the known feedback from TORC1 to AKT [86] raises concerns about unwanted upstream activation of the pathway in the presence of such inhibitors. mTOR catalytic site inhibitors that can inhibit both TORC1 and TORC2 are in development now. These should abrogate this feedback effect by inhibiting AKT Ser473 phosphorylation by TORC2, though as this may not prevent Thr308 phosphorylation, some AKT activity might remain.

Inhibitors of upstream regulators (ERBB2/3; FGFRs) and of other key pathways that are activated in UC, e.g., the MAPK pathway are also relevant. The use of trastuzumab in UC with gene amplification or high levels of overexpression of ERBB2 has been suggested [87], though any defects in the pathway below this would be predicted to lead to resistance. Similarly, EGFR inhibitors might be useful in certain cases. Proteins such as AKT that act lower down in the pathway appear to be more attractive targets.

It is clear that whilst relevant inhibitors are available or in development, it will be essential to have individual tumour-specific information in order to apply these rationally. This is particularly important in light of the current evidence that indicates non-redundancy of key molecular events affecting pathway members in UC. The complexity of feedback regulation of the pathway and its interactions with other pathways, particularly the MAPK pathway indicate that most successful treatments are likely to involve combined targeting of two or more pathways. Though the application of targeted agents will not be straightforward, the availability of well-characterised UC cell lines with known molecular profiles will greatly facilitate preclinical testing both in vitro and in vivo.

\section{Conclusions}

Recent studies have identified multiple molecular mechanisms by which the PI3K pathway is activated in bladder tumours. It is already clear that non-canonical functions of some pathway components may play a role in different tumour subgroups and a key objective is to elucidate what these are and to develop methods to classify tumour samples according to the status of all of these functions. This will allow patient selection for individualised targeted therapy, drawing on the wealth of novel agents that will soon be available.

Acknowledgements M.K, F.P and C.H are supported by Cancer Research UK (C6228/A5437) and R.R by a studentship from the Medical Research Council.

Open Access This article is distributed under the terms of the Creative Commons Attribution Noncommercial License which permits any noncommercial use, distribution, and reproduction in any medium, provided the original author(s) and source are credited.

\section{References}

1. Cantley, L. C. (2002). The phosphoinositide 3-kinase pathway. Science, 296, 1655-1657. 
2. Luo, J., Manning, B. D., \& Cantley, L. C. (2003). Targeting the PI3K-Akt pathway in human cancer: rationale and premise. Cancer Cell, 4, 257-262.

3. Shaw, R. J., \& Cantley, L. C. (2006). Ras, PI(3)K and mTOR signalling controls tumour cell growth. Nature, 441, 424-430.

4. Eng, C. (2003). PTEN: one gene, many syndromes. Human Mutation, 22, 183-198.

5. Launonen, V. (2005). Mutations in the human LKB1/STK11 gene. Human Mutation, 26, 291-297.

6. Philp, A. J., Campbell, I. G., Leet, C., Vincan, E., Rockman, S. P., Whitehead, R. H., et al. (2001). The phosphatidylinositol 3'-kinase p85alpha gene is an oncogene in human ovarian and colon tumors. Cancer Research, 61, 7426-7429.

7. Parsons, D. W., Wang, T. L., Samuels, Y., Bardelli, A., Cummins, J. M., DeLong, L., et al. (2005). Colorectal cancer: mutations in a signalling pathway. Nature, 436, 792

8. Samuels, Y., \& Ericson, K. (2006). Oncogenic PI3K and its role in cancer. Current Opinion in Oncology, 18, 77-82.

9. Carpten, J. D., Faber, A. L., Horn, C., Donoho, G. P., Briggs, S. L., Robbins, C. M., et al. (2007). A transforming mutation in the pleckstrin homology domain of AKT1 in cancer. Nature, 448, 439-444.

10. Ramjaun, A. R., \& Downward, J. (2007). Ras and phosphoinositide 3-Kinase: partners in development and tumorigenesis. Cell Cycle, 66(23), 2902-2905.

11. Ma, L., Chen, Z., Erdjument-Bromage, H., Tempst, P., \& Pandolfi, P. P. (2005). Phosphorylation and functional inactivation of TSC2 by Erk implications for tuberous sclerosis and cancer pathogenesis. Cell, 121, 179-193.

12. Yuan, T. L., \& Cantley, L. C. (2008). PI3K pathway alterations in cancer: variations on a theme. Oncogene, 27, 5497-5510.

13. Wu, X. R. (2005). Urothelial tumorigenesis: a tale of divergent pathways. Nature Reviews Cancer, 5, 713-725.

14. Knowles, M. A. (2006). Molecular subtypes of bladder cancer: Jekyll and Hyde or chalk and cheese? Carcinogenesis, 27, 361373.

15. Knowles, M. A. (2008). Bladder cancer subtypes defined by genomic alterations. Scandinavian Journal of Urology and Nephrology 42(S218t) 116-130.

16. Sarbassov, D. D., Guertin, D. A., Ali, S. M., \& Sabatini, D. M. (2005). Phosphorylation and regulation of Akt/PKB by the rictormTOR complex. Science, 307, 1098-1101.

17. Bozulic, L., Surucu, B., Hynx, D., \& Hemmings, B. A. (2008). PKBalpha/Akt1 acts downstream of DNA-PK in the DNA doublestrand break response and promotes survival. Molecular Cell, 30, 203-213.

18. Cappellen, D., Diez, G., de Medina, S., Chopin, D., Thiery, J. P., \& Radvanyi, F. (1997). Frequent loss of heterozygosity on chromosome $10 \mathrm{q}$ in muscle-invasive transitional cell carcinomas of the bladder. Oncogene, 14, 3059-3066.

19. Cairns, P., Evron, E., Okami, K., Halachmi, N., Esteller, M., Herman, J. G., et al. (1998). Point mutation and homozygous deletion of PTEN/MMAC1 in primary bladder cancers. Oncogene, 16, 3215-3218.

20. Aveyard, J. S., Skilleter, A., Habuchi, T., \& Knowles, M. A. (1999). Somatic mutation of PTEN in bladder carcinoma. British Journal of Cancer, 80, 904-908.

21. Wang, D. S., Rieger-Christ, K., Latini, J. M., Moinzadeh, A., Stoffel, J., Pezza, J. A., et al. (2000). Molecular analysis of PTEN and MXI1 in primary bladder carcinoma. International Journal of Cancer, 88, 620-625.

22. Zhao, L., \& Vogt, P. K. (2008). Helical domain and kinase domain mutations in p110(alpha) of phosphatidylinositol 3-kinase induce gain of function by different mechanisms. Proceedings of the National Academy of Sciences of the United States of America, $105,2652-2657$.
23. Platt, F. M., Hurst, C. D., Taylor, C. F., Gregory, W. M., Harnden, P., \& Knowles, M. A. (2009). Spectrum of phosphatidylinositol 3kinase pathway gene alterations in bladder cancer. Clinical Cancer Research, 15, 6008-6017.

24. Lopez-Knowles, E., Hernandez, S., Malats, N., Kogevinas, M., Lloreta, J., Carrato, A., et al. (2006). PIK3CA mutations are an early genetic alteration associated with FGFR3 mutations in superficial papillary bladder tumors. Cancer Research, 66, 7401-7404

25. Hurst, C. D., Zuiverloon, T. C., Hafner, C., Zwarthoff, E. C., \& Knowles, M. A. (2009). A SNaPshot assay for the rapid and simple detection of four common hotspot codon mutations in the PIK3CA gene. BMC Research Notes, 2, 66.

26. Samuels, Y., \& Velculescu, V. E. (2004). Oncogenic mutations of PIK3CA in human cancers. Cell Cycle, 3, 1221-1224.

27. Jebar, A. H., Hurst, C. D., Tomlinson, D. C., Johnston, C., Taylor, C. F., \& Knowles, M. A. (2005). FGFR3 and Ras gene mutations are mutually exclusive genetic events in urothelial cell carcinoma. Oncogene, 24, 5218-5225.

28. Billerey, C., Chopin, D., Aubriot-Lorton, M. H., Ricol, D., Diez, G., de Medina, S., et al. (2001). Frequent FGFR3 mutations in papillary non-invasive bladder (pTa) tumors. American Journal of Pathology, 158, 1955-1959.

29. Hafner, C., Lopez-Knowles, E., Luis, N. M., Toll, A., Baselga, E., Fernandez-Casado, A., et al. (2007). Oncogenic PIK3CA mutations occur in epidermal nevi and seborrheic keratoses with a characteristic mutation pattern. Proceedings of the National Academy of Sciences of the United States of America, 104, 13450-13454.

30. Gu, J., Tamura, M., Pankov, R., Danen, E. H., Takino, T., Matsumoto, K., et al. (1999). Shc and FAK differentially regulate cell motility and directionality modulated by PTEN. Journal of Cell Biology, 146, 389-403.

31. Planchon, S. M., Waite, K. A., \& Eng, C. (2008). The nuclear affairs of PTEN. Journal of Cell Science, 121, 249-253.

32. Kagan, J., Liu, J., Stein, J. D., Wagner, S. S., Babkowski, R., Grossman, B. H., et al. (1998). Cluster of allele losses within a $2.5 \mathrm{cM}$ region of chromosome 10 in high-grade invasive bladder cancer. Oncogene, 16, 909-913.

33. Liu, J., Babaian, D. C., Liebert, M., Steck, P. A., \& Kagan, J. (2000). Inactivation of MMAC1 in bladder transitional-cell carcinoma cell lines and specimens. Molecular Carcinogenesis, $29,143-150$.

34. Tsuruta, H., Kishimoto, H., Sasaki, T., Horie, Y., Natsui, M., Shibata, Y., et al. (2006). Hyperplasia and carcinomas in ptendeficient mice and reduced PTEN Protein in human bladder cancer patients. Cancer Research, 66, 8389-8396.

35. Puzio-Kuter, A. M., Castillo-Martin, M., Kinkade, C. W., Wang, X., Shen, T. H., Matos, T., et al. (2009). Inactivation of p53 and Pten promotes invasive bladder cancer. Genes and Development, 23, 675-680.

36. Di Cristofano, A., Pesce, B., Cordon-Cardo, C., \& Pandolfi, P. P. (1998). Pten is essential for embryonic development and tumour suppression. Nature Genetics, 19, 348-355.

37. Wu, X., Obata, T., Khan, Q., Highshaw, R. A., De Vere White, R., \& Sweeney, C. (2004). The phosphatidylinositol-3 kinase pathway regulates bladder cancer cell invasion. BJU International, 93, $143-150$.

38. Dey, N., Crosswell, H. E., De, P., Parsons, R., Peng, Q., Su, J. D., et al. (2008). The protein phosphatase activity of PTEN regulates SRC family kinases and controls glioma migration. Cancer Research, 68, 1862-1871.

39. Chiang, G. J., Billmeyer, B. R., Canes, D., Stoffel, J., Moinzadeh, A., Austin, C. A., et al. (2005). The src-family kinase inhibitor PP2 suppresses the in vitro invasive phenotype of bladder carcinoma cells via modulation of Akt. BJU International, 96, 416-422. 
40. Gildea, J. J., Herlevsen, M., Harding, M. A., Gulding, K. M., Moskaluk, C. A., Frierson, H. F., et al. (2004). PTEN can inhibit in vitro organotypic and in vivo orthotopic invasion of human bladder cancer cells even in the absence of its lipid phosphatase activity. Oncogene, 23, 6788-6797.

41. Yoo, L. I., Liu, D. W., Le Vu, S., Bronson, R. T., Wu, H., \& Yuan, J. (2006). Pten deficiency activates distinct downstream signaling pathways in a tissue-specific manner. Cancer Research, 66, 19291939.

42. Askham, J. M., Platt, F., Chambers, P. A., Snowden, H., Taylor, C. F., \& Knowles, M. A. (2009). AKT1 mutations in bladder cancer: identification of a novel oncogenic mutation that can co-operate with E17K. Oncogene (in press)

43. Gomez, M., Sampson, J., \& Whittemore, V. (1999). The tuberous sclerosis complex. Oxford University Press.

44. van Slegtenhorst, M., de Hoogt, R., Hermans, C., Nellist, M., Janssen, B., Verhoef, S., et al. (1997). Identification of the tuberous sclerosis gene TSC1 on chromosome 9q34. Science, 277, 805-808.

45. Consortium. (1993). Identification and characterization of the tuberous sclerosis gene on chromosome 16. The European chromosome 16 tuberous sclerosis consortium. Cell, 75, 13051315.

46. Plank, T. L., Yeung, R. S., \& Henske, E. P. (1998). Hamartin, the product of the tuberous sclerosis 1 (TSC1) gene, interacts with tuberin and appears to be localized to cytoplasmic vesicles. Cancer Research, 58, 4766-4770.

47. Johnson, M. W., Emelin, J. K., Park, S. H., \& Vinters, H. V. (1999). Co-localization of TSC1 and TSC2 gene products in tubers of patients with tuberous sclerosis. Brain Pathology, 9, 4554.

48. Hodges, A. K., Li, S., Maynard, J., Parry, L., Braverman, R., Cheadle, J. P., et al. (2001). Pathological mutations in TSC1 and TSC2 disrupt the interaction between hamartin and tuberin. Human Molecular Genetics, 10, 2899-2905.

49. Inoki, K., Li, Y., Xu, T., \& Guan, K. L. (2003). Rheb GTPase is a direct target of TSC2 GAP activity and regulates mTOR signaling. Genes and Development, 17, 1829-1834.

50. Tee, A. R., Manning, B. D., Roux, P. P., Cantley, L. C., \& Blenis, J. (2003). Tuberous sclerosis complex gene products, Tuberin and Hamartin, control mTOR signaling by acting as a GTPaseactivating protein complex toward Rheb. Current Biology, 13, $1259-1268$

51. Hornigold, N., Devlin, J., Davies, A. M., Aveyard, J. S., Habuchi, T., \& Knowles, M. A. (1999). Mutation of the 9q34 gene TSC1 in sporadic bladder cancer. Oncogene, 18, 2657-2661.

52. Adachi, H., Igawa, M., Shiina, H., Urakami, S., Shigeno, K., \& Hino, O. (2003). Human bladder tumors with 2-hit mutations of tumor suppressor gene TSC1 and decreased expression of p27. Journal of Urology, 170, 601-604.

53. Cairns, P., Shaw, M. E., \& Knowles, M. A. (1993). Initiation of bladder cancer may involve deletion of a tumour-suppressor gene on chromosome 9. Oncogene, 8, 1083-1085.

54. Habuchi, T., Devlin, J., Elder, P. A., \& Knowles, M. A. (1995). Detailed deletion mapping of chromosome $9 \mathrm{q}$ in bladder cancer: evidence for two tumour suppressor loci. Oncogene, 11, 1671-1674.

55. Simoneau, M., Aboulkassim, T. O., LaRue, H., Rousseau, F., \& Fradet, Y. (1999). Four tumor suppressor loci on chromosome 9q in bladder cancer: evidence for two novel candidate regions at $9 q 22.3$ and $9 \mathrm{q} 31$. Oncogene, 18, 157-163.

56. van Tilborg, A. A., Groenfeld, L. E., van der Kwast, T. H., \& Zwarthoff, E. C. (1999). Evidence for two candidate tumour suppressor loci on chromosome $9 \mathrm{q}$ in transitional cell carcinoma (TCC) of the bladder but no homozygous deletions in bladder tumour cell lines. British Journal of Cancer, 80, 489494.
57. Knowles, M. A., Habuchi, T., Kennedy, W., \& Cuthbert-Heavens, D. (2003). Mutation spectrum of the $9 \mathrm{q} 34$ tuberous sclerosis gene TSC1 in transitional cell carcinoma of the bladder. Cancer Research, 63, 7652-7656.

58. Pymar, L. S., Platt, F. M., Askham, J. M., Morrison, E. E., \& Knowles, M. A. (2008). Bladder tumour derived somatic TSC1 missense mutations cause loss of function via distinct mechanisms. Human Molecular Genetics, 17, 2006-2017.

59. Mozaffari, M., Hoogeveen-Westerveld, M., Kwiatkowski, D., Sampson, J., Ekong, R., Povey, S., et al. (2009). Identification of a region required for TSC1 stability by functional analysis of TSC1 missense mutations found in individuals with tuberous sclerosis complex. BMC Medical Genetic, 10, 88.

60. Nellist, M., van den Heuvel, D., Schluep, D., Exalto, C., Goedbloed, M., Maat-Kievit, A., et al. (2009). Missense mutations to the TSC1 gene cause tuberous sclerosis complex. European Journal of Human Genetics, 17, 319-328.

61. Henske, E. P., Scheithauer, B. W., Short, M. P., Wollmann, R., Nahmias, J., Hornigold, N., et al. (1996). Allelic loss is frequent in tuberous sclerosis kidney lesions but rare in brain lesions. American Journal of Human Genetics, 59, 400-406.

62. Cheadle, J. P., Reeve, M. P., Sampson, J. R., \& Kwiatkowski, D. J. (2000). Molecular genetic advances in tuberous sclerosis. Human Genetics, 107, 97-114.

63. Soltoff, S. P., Carraway, K. L., 3 rd, Prigent, S. A., Gullick, W. G., \& Cantley, L. C. (1994). ErbB3 is involved in activation of phosphatidylinositol 3-kinase by epidermal growth factor. Molecular and Cellular Biology, 14, 3550-3558.

64. Moriyama, M., Akiyama, T., Yamamoto, T., Kawamoto, T., Kato, T., Sato, K., et al. (1991). Expression of c-erbB-2 gene product in urinary bladder cancer. Journal of Urology, 145, 423-427.

65. Sato, K., Moriyama, M., Mori, S., Saito, M., Watanuki, T., Terada, K., et al. (1992). An immunohistologic evaluation of C-erbB-2 gene product in patients with urinary bladder carcinoma. Cancer, 70, 2493-2498.

66. Lipponen, P., \& Eskelinen, M. (1994). Expression of epidermal growth factor receptor in bladder cancer as related to established prognostic factors, oncoprotein (c-erbB-2, p53) expression and long-term prognosis. British Journal of Cancer, 69, $1120-1125$.

67. Chow, N. H., Chan, S. H., Tzai, T. S., Ho, C. L., \& Liu, H. S. (2001). Expression profiles of ErbB family receptors and prognosis in primary transitional cell carcinoma of the urinary bladder. Clinical Cancer Research, 7, 1957-1962.

68. Memon, A. A., Sorensen, B. S., Melgard, P., Fokdal, L., Thykjaer, T., \& Nexo, E. (2004). Expression of HER3, HER4 and their ligand heregulin-4 is associated with better survival in bladder cancer patients. British Journal of Cancer, 91, 2034-2041.

69. Memon, A. A., Sorensen, B. S., Meldgaard, P., Fokdal, L., Thykjaer, T., \& Nexo, E. (2006). The relation between survival and expression of HER1 and HER2 depends on the expression of HER3 and HER4: a study in bladder cancer patients. British Journal of Cancer, 94, 1703-1709.

70. Kassouf, W., Black, P. C., Tuziak, T., Bondaruk, J., Lee, S., Brown, G. A., et al. (2008). Distinctive expression pattern of ErbB family receptors signifies an aggressive variant of bladder cancer. Journal of Urology, 179, 353-358.

71. Jimenez, R. E., Hussain, M., Bianco, F. J., Jr., Vaishampayan, U., Tabazcka, P., Sakr, W. A., et al. (2001). Her-2/neu overexpression in muscle-invasive urothelial carcinoma of the bladder: prognostic significance and comparative analysis in primary and metastatic tumors. Clinical Cancer Research, 7, 2440-2447.

72. Kruger, S., Weitsch, G., Buttner, H., Matthiensen, A., Bohmer, T., Marquardt, T., et al. (2002). HER2 overexpression in muscleinvasive urothelial carcinoma of the bladder: prognostic implications. International Journal of Cancer, 102, 514-518. 
73. Cheng, H. L., Trink, B., Tzai, T. S., Liu, H. S., Chan, S. H., Ho, C. L., et al. (2002). Overexpression of c-met as a prognostic indicator for transitional cell carcinoma of the urinary bladder: a comparison with p53 nuclear accumulation. Journal of Clinical Oncology, 20, 1544-1550.

74. Cheng, H. L., Liu, H. S., Lin, Y. J., Chen, H. H., Hsu, P. Y., Chang, T. Y., et al. (2005). Co-expression of RON and MET is a prognostic indicator for patients with transitional-cell carcinoma of the bladder. British Journal of Cancer, 92, 1906-1914.

75. Sanchez-Carbayo, M., Socci, N. D., Lozano, J. J., Haab, B. B., \& Cordon-Cardo, C. (2006). Profiling bladder cancer using targeted antibody arrays. American Journal of Pathology, 168, 93-103.

76. Rieger-Christ, K. M., Cain, J. W., Braasch, J. W., Dugan, J. M., Silverman, M. L., Bouyounes, B., et al. (2001). Expression of classic cadherins type I in urothelial neoplastic progression. Human Pathology, 32, 18-23.

77. Rieger-Christ, K. M., Lee, P., Zagha, R., Kosakowski, M., Moinzadeh, A., Stoffel, J., et al. (2004). Novel expression of Ncadherin elicits in vitro bladder cell invasion via the Akt signaling pathway. Oncogene, 23, 4745-4753.

78. di Martino, E., L'Hote, C. G., Kennedy, W., Tomlinson, D. C., \& Knowles, M. A. (2009). Mutant fibroblast growth factor receptor 3 induces intracellular signaling and cellular transformation in a cell type- and mutation-specific manner. Oncogene (in press).

79. Woods, A., Johnstone, S. R., Dickerson, K., Leiper, F. C., Fryer, L. G., Neumann, D., et al. (2003). LKB1 is the upstream kinase in the AMP-activated protein kinase cascade. Current Biology, 13, 2004-2008.
80. Shaw, R. J., Bardeesy, N., Manning, B. D., Lopez, L., Kosmatka, M., DePinho, R. A., et al. (2004). The LKB1 tumor suppressor negatively regulates mTOR signaling. Cancer Cell, 6, 91-99.

81. Sommerhaug, R. G., \& Mason, T. (1970). Peutz-Jeghers syndrome and ureteral polyposis. JAMA, 211, 120-122.

82. Sanchez-Cespedes, M. (2007). A role for LKB1 gene in human cancer beyond the Peutz-Jeghers syndrome. Oncogene, 26, 78257832.

83. Adley, B. P., Smith, N. D., Nayar, R., \& Yang, X. J. (2006). BirtHogg-Dube syndrome: clinicopathologic findings and genetic alterations. Archives of Pathology and Laboratory Medicine, 130, 1865-1870.

84. Engelman, J. A. (2009). Targeting PI3K signalling in cancer: opportunities, challenges and limitations. Nature Reviews Cancer, 9, 550-562.

85. Liu, P., Cheng, H., Roberts, T. M., \& Zhao, J. J. (2009). Targeting the phosphoinositide 3-kinase pathway in cancer. Nature Review Drug Discovery, 8, 627-644.

86. O'Reilly, K. E., Rojo, F., She, Q. B., Solit, D., Mills, G. B., Smith, D., et al. (2006). mTOR inhibition induces upstream receptor tyrosine kinase signaling and activates Akt. Cancer Research, 66, $1500-1508$

87. Hussain, M. H., MacVicar, G. R., Petrylak, D. P., Dunn, R. L., Vaishampayan, U., Lara, P. N., Jr., et al. (2007). Trastuzumab, paclitaxel, carboplatin, and gemcitabine in advanced human epidermal growth factor receptor-2/neupositive urothelial carcinoma: results of a multicenter phase II national cancer institute trial. Journal of Clinical Oncology, $25,2218-2224$. 